\title{
Corrigendum
}

\section{Corrigendum to "Numerical Analysis of the Coupling between Hydrogen Diffusion and Mechanical Behavior near the Crack Tip of Titanium"}

\author{
Fu-Qiang Yang $\mathbb{D}^{1},{ }^{1}$ Wen-Juan Zhang $\mathbb{D},{ }^{2}$ Tao Yan $\mathbb{D}^{2},{ }^{2}$ Hai-Bing Zhang $\mathbb{D}{ }^{3}$ \\ and Xiu-Rong Fang $\mathbb{B D}^{2}$

\footnotetext{
${ }^{1}$ School of Science, Xi'an University of Science \& Technology, Xi'an 710054, China

${ }^{2}$ School of Mechanical Engineering, Xi'an University of Science \& Technology, Xi'an 710054, China

${ }^{3}$ State Key Laboratory for Marine Corrosion and Protection, Luoyang Ship Material Research Institute (LSMRI), Qingdao 266237, China
}

Correspondence should be addressed to Fu-Qiang Yang; yang_afreet@163.com and Hai-Bing Zhang; zhanghb@sunrui.net

Received 22 July 2020; Accepted 23 July 2020; Published 12 October 2020

Copyright (c) $2020 \mathrm{Fu}$-Qiang Yang et al. This is an open access article distributed under the Creative Commons Attribution License, which permits unrestricted use, distribution, and reproduction in any medium, provided the original work is properly cited.

In the article titled "Numerical Analysis of the Coupling between Hydrogen Diffusion and Mechanical Behavior near the Crack Tip of Titanium" [1], there was a spelling error in author Wen-juan Zhan's name in the authors' list, where "Wen-juan Zhan" should have read "Wen-juan Zhang."

The corrected authors' list is shown above.

\section{References}

[1] F. Yang, W. Zhang, T. Yan, H. Zhang, and X. Fang, "Numerical analysis of the coupling between hydrogen diffusion and mechanical behavior near the crack tip of titanium," Mathematical Problems in Engineering, vol. 2020, Article ID 3618589, 15 pages, 2020. 